\title{
Preliminary Understanding of "Static" and the Homeostasis
}

\author{
Yue $\mathrm{Li}^{1 \mathrm{a}}$ \\ Department of psychology, Chengde Medical College, \\ Chengde, 067000, China
}

\author{
Ruiyu $\mathrm{Li}^{2}$, b,* \\ Institute for Integrated Traditional and Western Medicine, \\ Second Affiliated Hospital of Xingtai Medical College, \\ Xingtai, 054000, China \\ Liruiyu651021@163.com
}

\author{
Meng $\mathrm{Li}^{\mathrm{c}}$ \\ Health Team, Hotan Detachment of the Xinjiang Armed Police Corps \\ Hotan, 848011, Xinjiang Uygur Autonomous Region, China
}

\begin{abstract}
With the improvement of the standard of living, work pressure is also increasing today. People's tension, depression, anxiety, fear, depressed, upset, anger and other mood disorders do not reduce but become more frequent and intense with the social development. The author believes that people with those mood disorders have lost their due "static" scope and have close relationship with the range beyond "jing". In the process of social civilization construction, the integration of the situations of these problems is the focus of current research. Traditional medicine has always attached great importance to the study of preserve one's health. Its important academic thought can be summarized as "static", and "static" has close relationship with homeostasis, this article preliminary summary what is static? What is homeostasis? Social progress and human harmony need to be static and homeostasis.
\end{abstract}

Keywords-Steady; Steady state; Technology progress; Publisher; Designing; Lowercase letters

\section{INTRODUCTION}

In living standards improve, work pressure is also growing today, people's tension, depression, anxiety, fear, be agitated, depressed, upset, angry, such as mood disorders, can even appear sudden death, these phenomenon did not reduce along with the social development in many groups of people are more frequent, more intense [1-2].These phenomena occur in the author's opinion that people have lost their due "static" scope and have close relationship with the range beyond "jing".Currently, health care is becoming an important issue in people's life [3-6].Traditional Chinese medicine has always attached importance to the research on health preservation, and its important academic thought can be summarized as "jing".Jing "is of great significance to social life and psychology [7]. Meanwhile," jing "is closely related to the homeostasis of the human body. Social science and technology progress and harmony need to be" static "and stable in human body. What is static in this article? What is homeostasis? Static and homeostasis? Social progress and human harmony need a preliminary conclusion:

\section{WHAT IS STATIC}

For static, refer to the relevant explanation: jing, jing, from qing dynasty. Original meaning: Proper color distribution. With a net. Green, early biological color; Fight, both hands in the direction of both hands, persistence. Still, do not suffer from external disturbance and adhere to the original nature, adhere to the original. Yue jing. "Six towers" is a collection of all things. In winter, everything is quiet. Basic explanation: static, stop, inactive, and "move" relative: static. Static. Still life. Calm down. Calm and calm. No voice: silence. Silence. Quiet. Calm down. Silence. Quiet. Delivery. Quiet. Silence, contemplation, listening. Calm, quiet, quiet. Sit quietly. Quiet. Commonly used words: quiet, quiet, quiet, quiet zhiyuan, mountain long quiet day and night, static: refers to in the process of qigong exercise, in the quiet, focus on the basis of a sober, keep the mind is single-minded, the practise a state of relaxed and comfortable." The word "quiet", in the late song and early yuan dynasty, "History As A Mirror", tang xizong guangqi three years, said:" into the quiet, the quiet room, the screen left and right, clear and quiet, unselfish." Silence is also called idleness, as in the life of the king. Nothing in my mind is empty, I can't think of a quiet mind." It is similar to the "naturalization" of buddhist practice. When it comes to meditation, the mind does not go away and goes into a quiet state of meditation, namely, "into meditation", so it is also called "meditation". It is to ask for the distraction of the mind, through a certain amount of merit, set in one place. We believe that "jing" is the manifestation of unity, harmony and ease of mind. 


\section{WHAT Is HOMEOSTASIS?}

Homeostasis is a dynamic equilibrium within the body. By studying the sympathetic nervous system and related endocrine functions, cannon has a deeper and more detailed understanding of Bernard's internal environmental theory. In 1932, he put forward the theory of homeostasis in "wisdom of the human body". The term homeostasis describes the selfregulating process of maintaining a stable internal environment He proposed that the stability of the internal environment was not isolated from the environment, but by constantly regulating various physiological processes in the body. Steady state is a dynamic equilibrium that is not constant; the components are constantly changing and the whole system is stable. Although kanon realized that the homeostasis of the internal environment is the result of neuroendocrine and blood cushioning, its specific mechanism still needs to be further explored. In his later years, cannon and Henderson were convinced that life could not be fully divided into physical and chemical processes, that is, the structure and interaction of the parts of the life system and the simple physical and chemical processes. He sees the organism as a whole, each part having its own function, but integrating it through various control processes. This reflects an old and fashionable proposition: the whole is greater than the sum of the parts. People in body has returned to normal after the process is an example of homeostasis. Homeostasis is the body's internal can keep a dynamic equilibrium, namely, no matter how the external environment of an organism in vivo environment always remained stable.

Homeostasis is a more progressive mechanism in the process of evolutionary development. The organisms with homeostasis are relatively independent of external conditions by means of the stability of the internal environment. The homeostasis mechanism greatly improves the tolerance of biological factors to ecological factors. The theory of homeostasis is generally regarded as the sign of the establishment of modern physiology, and it is also the basis for the further development of physiology. After entering the 20th century, the development of physiology appeared two intense areas: neurophysiology and endocrine physiology.

\section{THE RELATIONSHIP BETWEEN STATIC AND HOMEOSTASIS.}

"Static" is not fixed, the "static" is relative to "static", is not absolute, is human body science system "static", "static" of natural, harmonious "static", "static" is the inner need. In a sense, it is also the "quiet" in motion. Static for everyone, we believe that the individual differences due to the human body, the work, the environment, career, interests, pursue different, so there may be different to static adaptation range, this more or less different expression in human brain consciousness and behavior change. But in any case, everyone should be positive, happy, positive and happy in their "quiet" best range, and everyone should pursue their goals. The optimal range of "static", we believe that the homeostasis of the whole body is the system coordination constant. Dynamic and static is the two basic forms of material existence, if the concept is about integrity, unity, is more static, so, the view of perpetual motion with movement to analyze and research the problem, more is moved. The harmonious view is to achieve the unity of the whole through the internal coordination movement, which can be described as dynamic and static combination. The homeostasis involves the regulation of every organ, tissue and cell activity in the body, and is represented at all levels from the cell to the overall biological system. Cells separated by the cell membrane and its surrounding environment, the cell interior changes and the liquid around the cell is very different, the cells and the surrounding liquid continuous material exchange and maintain its internal stability, which is the cell homeostasis. Maintaining a steady state of the whole body depends on the integration of hormones and nervous systems in higher animals. The hormone keeps the body steady, and it has the ability to release and stop hormones in time, which is the steady state of the hormone secretion. The central nervous system plays an important role in maintaining the homeostasis of the body. The steady state of the central nervous system depends on the constancy of the internal environment in which it is exposed. The 2017 Nobel Prize in physiology or medicine is awarded to American scientists Jeffrey c. Hall, Michael Rosbash and Michael w. Young. The winning reason was "the discovery of molecular mechanisms regulating circadian rhythms". Press conference of the Nobel Prize evaluation: "the discovery after released, circadian biology has developed into a broad, highly active area of research, have important implications for people's health and well-being. All creatures have a biological clock. The biological clock regulates $10 \%-$ $40 \%$ of the genes. It also affects various physiological processes such as blood pressure, blood sugar, sleep, metabolism and all sorts of psysiological processes. "No system is as generous as a circadian clock gene." Therefore, it is important to clarify the intrinsic mechanism of the biological clock. It is the model of basic scientific research, and the appearance of the biological clock enables the body to predict the changes of the environment, maintain the steady state of the body, and improve the survival ability. Three scientists through basic research concluded that clock disruptions, such as staying up late to destroy the balance of human body biological clock, will produce disease, can cause the key to adjust the rhythm genes expires, promotes tumorigenesis.

"Static" and homeostasis is harmonious and unified, if its own rule has been broken, beyond the scope of the body's own adjustment "static", then there is the best range of "static", such as: when the need to "static" sleep deprivation, sleep deprivation is what? Refers to the state of sleep loss due to various reasons, generally refers to total sleep within $24 \mathrm{~h}<4 \mathrm{~h}$ and cognition, learning, memory, mental, and immune function $\sim$ series such as physical and mental state and behavior change [9].

Recently, other studies have been conducted on the effects of functional magnetic resonance imaging of Fmri on brain function in sleep deprivation. Vartanian etc. [10] by sleep deprivation on the completion of tasks of the human brain divergent thinking fluency studies confirm that sleep deprivation makes the prefrontal cortex, impaired brain function activities, in turn, affects the brain's divergent and creative thinking. Klumpers et al [11] tested the speed of word recognition in healthy subjects after sleep deprivation and performed fMRI scan, the results showed that the group recognized the accuracy of neutral and positive words, and the 
activation of the dorsolateral prefrontal cortex was enhanced, while the activation of island leaves was enhanced when the emotion words were identified. In addition, the scholars also introduced a number of objective indicators, such as mental mood related scales, PET/CT data and salivary cortisol, to analyze the effects of sleep deprivation on the physiological function of human brain. Other scholars have analyzed the static and resting brain function of sleep deprivation from the level of brain function network. Gujar et al. [12] confirmed that the default mode network of the human brain after sleep deprivation was damaged by different degrees. Wang et al. [13] performed resting fMRI scanning on 23 healthy subjects after sleep deprivation. The results showed that the functional connectivity between the two submodules in the default mode network decreased. The results also confirmed that the mental and emotional states of the subjects were worse. The degree of reduction in functional connectivity is more serious.

In addition, the "static" range of disorders can make love to the disease, "emotional illness" is an important part of the basic theory of Chinese medicine psychology. Modern medical research, when emotional anger, sympathetic nervous system is activated, sympathetic adrenal medullary system causes excitement; Adrenocortical hormone, renin angiotensin, thyroxine, glucagon and pituitary hormone secretion increased after the endocrine system activated. These hormones change. It is mainly caused by the imbalance of NEI system. Prolonged exposure to this state of stress can result in a poor body response, which can affect the body's neuroendocrine system and immune system. Appropriate exercise training improves the adaptability of the system. Reducing the secretion of endogenous stress hormone levels may be one of the important mechanisms for the influence of exercise on the neuroimmune system [14]. Depression patients have chronic long-term excitations of the hypothalamus pituitary adrenal axis, leading to immune dysfunction. At the same time, some research system of complex disease medical perspective: endogenous network theory, using functional landscape to understand complex disease development, made to validate the new prediction for complex disease prevention, diagnosis, treatment to provide a series of potential ideas and solutions [15].

In a word, "static" is closely related to the homeostasis, people live and work in process, keep the "static" agree with homeostasis, can make human body health performance [16], otherwise may have bad side, the impact performance in many ways [17], neijing "sick from using" view of the "grasping with the dual nature of the disease", "keep fit prevention theory based on the close" and "medicine sent with flat for the prudential" etc [18]. have important significance. "Sick from using" talk about neijing nonequilibrium steady state, with a steady and orderly (physical) lost a steady disorder (pathology) model to explain TCM pathogenesis view, and maybe more accord with the onset of neijing learning thought [19].

\section{Social SCIENCE AND TECHNOLOGy PROgRESS AND HARMONY NEED TO BE "STATIC" AND HOMEOSTASIS}

As you know, professor huang is a full-time professor at the earth exploration science and technology Institute of Jilin University, a special expert of the national "thousand talents program", and the introduction of talents in key disciplines of the ministry of education. He worked hard and worked hard all day long. He died of illness on January 8, 2017. He is indifferent to fame and wealth, assiduously, dedicated noble sentiment, the patriotism, patriotic ambition is incorporated in the great cause of reform and development of the motherland, is incorporated in the great struggle of the people make history. During his seven years in China, huang led more than 400 scientists to create a number of "China's first", and filled a number of technical gaps for our country "to explore the land". With the ninth project he is responsible for - the conclusion of the research and experiment on key instruments and equipment for deep exploration, the achievements of China's "deep exploration technology and experimental research" project for 5 years have exceeded the past 50 years and the deep exploration capability has reached the international First-class level and local leading position in the world ... "China is going to be a great power," he said. "There needs to be a number of 'scientific lunatics'. The motherland needs to teach the talents of the professor.

Both on the hand, professer huang needs to be quiet, if he can be quiet, there would be no result of January 8, 2017. What we mean by "static" is not to say that we are not working or not exercising. But does not exceed the scope of the body's own regulation, the work, the body, cause together to maintain a balanced internal stability. The body is a revolutionary capital, no body, no one, no matter how good the cause itself can not continue, more need to do the work can not work on themselves, on the state is a big loss, for themselves, is a loss of life. It is the loss of talent for the country, the loss of the world for the advancement of human scientists.

If it's "quiet", the homeostasis is relatively balanced. Homeostasis balance, Chinese medicine called "yin-yang Yang secret," naturally no serious illness. In the Ming dynasty, "the four needs of health": "the heart is always quiet and the god is safe, and the spirit of god is good, so that life is not dangerous." Speak of traditional Chinese medicine with god, and god, refers to the human body and spirit, is a pair of the structure of the human body and the category of nature and life relationship [20], god phase to reach steady state of life, namely phase from the shape and break the life steady [21]

Therefore, "static" and homeostasis are important to human body, which should be taken seriously at the national level, and corresponding protective measures should be formulated, "static" and homeostasis are effective for human body. The lesson is painful, need to summarize, need method, need prevention and cure. If a repeat, to promote and encourage, again good spirit to encourage, such incentives and spiritual if not before things do a good job in the early stage, there could be more like professor Huang Danian results by scientists in order to serve the motherland, again good spirit to encourage, such incentives and spiritual if not before things do a good job in the early stage of the three could be more like professor 
Huang Danian results by scientists in order to serve the motherland. Therefore, no matter how society fast development, the human factor is always the first, human resource development, innovation power, is not just a slogan, but also the human factor relates to the steady state in the human body and "static", let them have a healthy body, a greater age,. In some sense, they sometimes do not represent themselves. They represent the motherland and represent the progress of science and technology. They aspire to wealth and create wealth. They undertake civilization and promote civilization. Edison said, "Genius is equal to ninety-nine percent perspiration and one percent inspiration." The inspiration of these scientists is priceless, and these ideas are not replicable., Knowing the inspirational importance of these scientists, then you can better understand and value their values, handle well, make good decisions and implement relevant policies so that these scientists In a good "static" to maintain the body's steady state environment, so that technology innovation can be long-term, but also a symbol of social progress and harmony.

\section{CONCLUSION}

The world health organization (WHO) defines a health definition as: health is not only free of disease, but also includes four aspects: physical health, mental health, social adaptation and moral health. Through the author's analysis, in the process of our country's social development, along with the improvement of productivity and scientific and technological progress, it brings people many psychological barriers and even suicide. It is discussed that these phenomena may be lost due to "static" range, resulting in "homeostasis" imbalance, which is beyond the "static" range, resulting in "homeostasis" imbalance, and many scientific and technical personnel are working hard. This paper highlights the importance of "static" and homeostasis to human body, which is of great significance to people's work, life and scientific and technological progress.

\section{REFERENCES}

[1] Xiao Hanshi, He Daren, Xiao jing, etc., On the impact of modern socia changes on people's mental health, medicine and philosophy, Vol. 26 No. 4, pp. 67,10 2005

[2] Peng Ruxin, Miao Weili, Zhou Shijin, Chaotic medicine, steady state and sudden death, Chinese tissue engineering research and clinica rehabilitation, Vol. 12, No. 15, pp. 2956-2960, 2008.

[3] Gao Xiaojing, Liu Shaoyun, Deng Qiwei, The spiritual psychological conditioning method in the huangdi neijing, the national medicine of Chinese medicine.Vol. 20, No. 1, pp. 236-237,2009.

[4] Li Dongnan, Health theory of traditional Chinese medicine, Journal of Hubei University of Chinese Medicine, February 2017, Vol. 19, No. 1, pp. 51-53.

[5] LI Xiaofang, LI Yajun, Discussion on Application Prospect of the Phase of the Moon and the Changes in Nei Jing in the Aspect of Health Preserving, Aug. 2016, Vol. 40, No. 4, pp.45-47.

[6] LI En, LI Zhaoguo, LI Zhenjiang, Yellow Emperor's Canon of Medicine: Theoretical Development and Modern Studies (II), Beijing: China traditional Chinese medicine press, pp. 400, 2016.

[7] Zhuang Xiaowei, Chen Yuming, Liu han, et al., mental health and daily life activities of the elderly, cross-sectional survey of social support, Chinese Journal of Rehabilitation Medicine, Oct. 2017,V01. 32,No. 10, pp. 1162-1166, 2017.

[8] Ren Pingjun, Li Ruiyu, The harmonious view of Chinese medicine, modern Chinese and western medicine journal, Vol.20, No.27, pp.34443446, 2011.

[9] Davies SK, Ang JE, Revell VL, et a1. Effec t of sleep deprivation on the human metabolom. Proc Natl Acad Sci U S A, 2014, 111 (29):10761. 10766

[10] Vartanian O, Bouak F, CaldwellJL, et a1. The effects of a single night ofsleep deprivation on fluency and prefrontal cortex function during divergent thinking. Front Hum Neurosci, 2014, 8(1):214.

[11] Klumpers UM,Veltman DJ,Van Tol MJ,et a1. Neurophysiological effects of sleep deprivation in healthy adults, a pilo study. PLoSOne,2015,10(I):eO116906.

[12] Gujar N, Yoo SS, Hu P, et a1. The unrested resting brain: sleep deprivation alters activity within the default-mode network. J CognNeurosci, 2010, 22(8):1637-1648.

[13] Wang $Y^{\prime}$ Liu H, Hitchman G, et a1. Module number of default modenetwork: inter-subject variability and effects of sleep deprivation. Brain Res, 2015, 1596(1):6.

[14] YIN Jianchun, Yan jun, Research on Effects of Exercise Training on Several Indexes of Neuroimmune System of Rats after Mental Stress, JournaI of Beijing Sport University, vol. 29, no. 1, pp. 70, 2006.

[15] Su hang, Wang Gaowei, Zhu Xiaomei, etc., System medical perspective of complex diseases: endogenous network theory, Chinese Journal of Nature, Vol. 37, No. 6, pp.448-454, 2015.

[16] Steady state medical view (on), Chinese J Basic Med Tcm, vol. 7, no. 10 pp. 19-21, 2001

[17] Liu Chengyuan, luo Hongyan, Zhang Rongli, Steady state medical view (below), Chinese J Basic Med Tcm, Vol. 7, No. 11, pp. 2-4, 2001.

[18] Qian Huinan, From neijing "sick with" parsing, Tcm Res. vol. 15, no. 2, 2002.

[19] Wen Yuezhang, Zhang Zengjia, From "illness from the use of" to talk about the non-equilibrium state of the mind, Jiangxi Chinese medicine, Vol. 41, No. 7, pp.11-13, 2009.

[20] Xue Gongchen, On the Confucian Buddha in medicine. Beijing: Traditional Chinese medicine publishing house.pp.287, 1999

[21] Lv ling, Lu xiulan, Tan Sujuan, The theory of the steady-state view of Chinese medicine, JTCM. May 2006, V01. 47, No. 5 pp.326-328. 